Article

\title{
Advanced Wireless Sensor Networks for Sustainable Buildings Using Building Ducts
}

\author{
Heekwon Yang ${ }^{1}$, Byeol Kim ${ }^{2}$ (D) , Joosung Lee ${ }^{2, *}$, Yonghan Ahn ${ }^{2}$ and Chankil Lee ${ }^{1}$ \\ 1 Department of Electronics and Communications Engineering, Hanyang University, ERICA Campus, \\ Ansan 15588, Korea; hkyang.econnect@gmail.com (H.Y.); cklee@hanyang.ac.kr (C.L.) \\ 2 Department of Architectural Engineering, Hanyang University, ERICA Campus, Ansan 15588, Korea; \\ keemstars@naver.com (B.K.); yhahn@hanyang.ac.kr (Y.A.) \\ * Correspondence: neowings@naver.com
}

Received: 30 May 2018; Accepted: 23 July 2018; Published: 26 July 2018

\begin{abstract}
The communication technology ZigBee has been widely adopted in wireless sensor networks (WSNs) for a wide range of industrial applications. However, although ZigBee provides low-power, low-cost mesh networking, it cannot guarantee steady and predictable network performance as channels are time-variant and highly attenuated by man-made obstacles. The networks also suffer from interference, especially in the important $2.4 \mathrm{GHz}$ industrial, scientific, and medical (ISM) band. These degraded channel characteristics increase the number of hops, thus increasing both the packet error rate and transmission delays. In this paper, we report the deployment of a ZigBee-based WSN inside an existing building duct system utilized for intelligent waste collection in an industrial environment. The Received Signal Strength (RSS) and path losses were measured, revealing that the duct communication channel acts as a very effective waveguide, providing a more reliable and consistent network performance than conventional free space channels.
\end{abstract}

Keywords: duct communication; wireless sensor network; ZigBee; industrial automation; automatic waste collection system; EM35; intelligent building system; sustainable building

\section{Introduction}

Wireless sensor networks (WSNs) are becoming popular for a wide range of applications in countries around the world due to their effectiveness in minimizing resources, their low installation costs, their environmental cleanliness, and their many social benefits, thus contributing to the three dimensions of sustainability: economic, environmental, and social [1]. As a result, the so-called Internet of Things (IoT) is expanding into every aspect of our lives, including sectors such as health, education, enterprises, and industries that are vital for our well-being, increasing their value in terms of their initial/functional costs, energy usage, and quality management [2,3]. WSNs can offer convenient and cost-effective solutions by providing data from sensors and controlling industrial applications [4-12]. However, conventional deployments for indoor wireless communications often suffer from unpredictable and variable attenuation for a number of reasons, as explained by Gungor and Hancke [13]. These problems may dilute the benefits of WSN, namely low installation costs and network expandability, compared to the more reliable wired networks. Among other issues, intervening structures and obstructions in buildings such as walls, partitions, and moving objects tend to introduce interference and propagation loss, making the system reliability poor [14]. WSNs operating in the $2.4 \mathrm{GHz}$ ISM (industrial, scientific, and medical) band may also be coexisting with Bluetooth or $\mathrm{Wi}-\mathrm{Fi}$, causing further interference in the network [15].

The path loss between two communicating nodes depends upon the medium in which they are operating and the radiation pattern of the two communicating antennas. This is particularly severe in 
industrial environments, where the propagation is strongly influenced by features such as the layout of the building, the types of machinery present, and the construction material and building type. Signal levels depend on factors as seemingly trivial as the room door positions (i.e., open or closed) as well as the sensor nodes' geographical positions and line-of-sight (LOS). A typical WSN incurs indoor partition losses, path losses, attenuation, poor signal penetration into the building, and partial transmission losses due to reflections from surfaces.

Unfortunately, wireless communication technologies are critical components of intelligent building systems, which incorporate a great deal of IoT equipment, primarily sensors and actuators. In an intelligent building, each room and space has its own role to play, which may involve diverse sensors and actuators that support indoor lighting, illumination levels, thermostat settings, AMI (Advanced Metering Infrastructure)/DR (Demand Response), human activity and fire detection, RFID readers, and so on and so forth. Viewed in this light, any reduction in the sensitivity of a building's wireless network will have a serious impact on its intelligent building systems.

In order to increase the wireless network system reliability, these constraints must be addressed. One approach is to change the medium to one with a much lower propagation loss. This not only reduces the propagation loss significantly but also eliminates any interference from coexistent technologies like Bluetooth and Wi-Fi. However, there are already multiple low-loss propagation channels installed in almost every modern industrial building and area in the form of heating, ventilation, and air-conditioning (HVAC) ducts, garbage collection pipes, and gas pipelines. Networks of these hollow pipes, which effectively act as waveguides, have been shown to act as very reliable communication channels for the propagation of intra-building communications [16]. Such networks not only avoid interference from outside networks but also provide a reliable propagation channel with much lower path loss and attenuation than free space. Xhafa et al. [17] demonstrated that HVAC ducts can indeed provide useful pathways for networks using the ISM band of $2.4 \mathrm{GHz}$, thus providing high quality internet access for indoor wireless networks.

In the work reported here, the potential utility of using hollow pipes originally installed for the management of solid waste (MSW) in high-rise buildings as a communication medium for a low cost, low data rate wireless ZigBee network is evaluated. Utilizing existing automatic waste collection system (AWCS) ducts as a wireless duct area network (WDAN) combines the advantages of a safe and reliable communications channel with efficient and environmentally friendly garbage disposal. Waste collection inside industrial, commercial, and residential high-rise buildings is often a challenge, especially in densely populated urban areas. The environmental impact caused by MSW has received a great deal of attention from both social and environmental viewpoints in recent decades, particularly in big cities in developing countries [18]. In some locations, up to $85 \%$ of a company's total expenditure may be devoted to waste collection and transportation, hence any improvement in the design of collection systems could result in significant savings [19]. To address this problem while at the same time supporting a green environment and sustainable development, state-of-the-art technology may prove to be part of the solution. Integrating a WDAN into a building's existing automatic waste collection processes can also increase the reliability of the company's communication systems.

The remainder of this paper is organized as follows. The next section presents a comprehensive literature review that describes recent research on both wired or wireless sensor networks in buildings and identifies the problems involved. Section 3 discusses the proposed approach for wireless communication networks in industrial settings and provides a detailed description of the analysis of the proposed new communication channel. A description of the experiments performed on a real-world duct communication implementation and the results obtained are presented in Section 4 . The paper concludes by summarizing the work, its implications for practice, its contribution to the field, and its novelty in Section 5. 


\section{Background and Literature Review}

This section presents a survey of existing work on building automation systems (BAS) that use both wired and wireless networks. WSN characteristics related to communication between nodes and their propagation have been studied by several researchers. For example, Suryadevara et al. [20] developed smart monitoring and control systems for household electrical appliances that use wireless networks, finding that this low-cost, flexible option saves electricity and thus reduces household expenses for consumers. However, the use of unlicensed radio frequency bands may cause channel interference between these and other nearby devices [21]. Langhammer and Kays [22] suggested guidelines to help decision makers develop suitable wireless standards for smart homes. Unlike wired solutions, wireless communication technologies are highly flexible and can be easily retrofitted in most buildings [23,24]. Wireless technologies do not require direct access to electrical installations, which may not be readily available in devices like outdoor sensors or gas meters. However, wireless technologies do suffer from the problem of propagation attenuation due to artificial or natural obstacles (free-space) and inner walls (indoors) [25]. Bhatt and Verma [24] considered home security and building automation systems that utilized wired networks. They concluded that in a wired network, actions such as adding, changing, and replacing sensors and actuators or reconfiguring, expanding, or upgrading the network incur significant additional costs. If an operator wishes to provide additional services, a service-specific network must be established. There is consequently a great deal of time and money involved in installing communication networks.

The use of WDAN has been suggested as potentially offering a very effective method for addressing the problems associated with both wireless and wired networks [26,27]. Xhafa et al. [17] pointed out that using an existing HVAC duct system has several advantages for signal distribution within buildings: (1) the HVAC duct system is already present in many buildings, (2) HVAC duct channels provide a time-invariant environment that is electrically shielded from outside interference, (3) the path loss in a straight HVAC duct is lower than that in a coaxial cable due to free-space propagation, and (4) multiple transmitting powers or frequencies can potentially be used inside HVAC ducts without violating emittance and interference regulations, provided that leakage from the duct system into the building space is negligible or can be controlled [28]. The previous work in this area therefore suggests that WDANs can sidestep many of the problems and limitations of wired networks while simultaneously supporting the three major sustainability goals related to economic, environmental, and social factors.

Almost all industrial buildings are constructed with a complex infrastructure consisting of HVAC ducts, pipes carrying waste, and gas pipelines, all of which are essentially waveguides. These pipes cover the whole building space, including offices, workshops, toilets, and corridors, representing a potentially invaluable resource for implementing a wireless communication system without the need to introduce additional wireless routers and other equipment to create an IoT communication network for the building's automation systems (BAS).

Figure 1 depicts a typical BAS network system implemented in an intelligent building. Almost all of the existing sensor network is connected with direct digital control (DDC) via cables, incurring many of the problems described earlier. In this paper, we propose the installation of a wireless sensor network within the building's existing duct system. Figure 2 shows our proposed architecture for deploying WDAN inside existing ducts/hollow pipes in a factory or building environment. These waveguides can provide a safe communication channel for all types of wireless communications. In the proposed architecture, sensor and/or actuator (SA) nodes are mounted inside a hollow pipe at some distance from each other, as shown in the figure. Each SA node is equipped with a radio control mode (RCM) for monitoring specific actions and consequently controlling the system wirelessly. These SA nodes are connected via a gateway $(\mathrm{G} / \mathrm{W})$ to the remote server, typically an internet-equipped personal computer or mobile handset. The remote server is equipped with a specially designed software tool for monitoring and control known as man-machine interface software (MMIS). In order to connect SA nodes to MMIS, the G/W device provides protocol translation from WDAN to wireline (TCP/IP, PLC) 
or wireless (WPAN, WLAN, WAN) network depending upon the application. Additional SA nodes, acting as either repeaters or routers, can be added as needed to increase the coverage area of a specific WDAN. This type of WDAN architecture will improve the reliability of a wireless building network system by enhancing the survivability coefficient of the radio waves travelling between SA nodes.
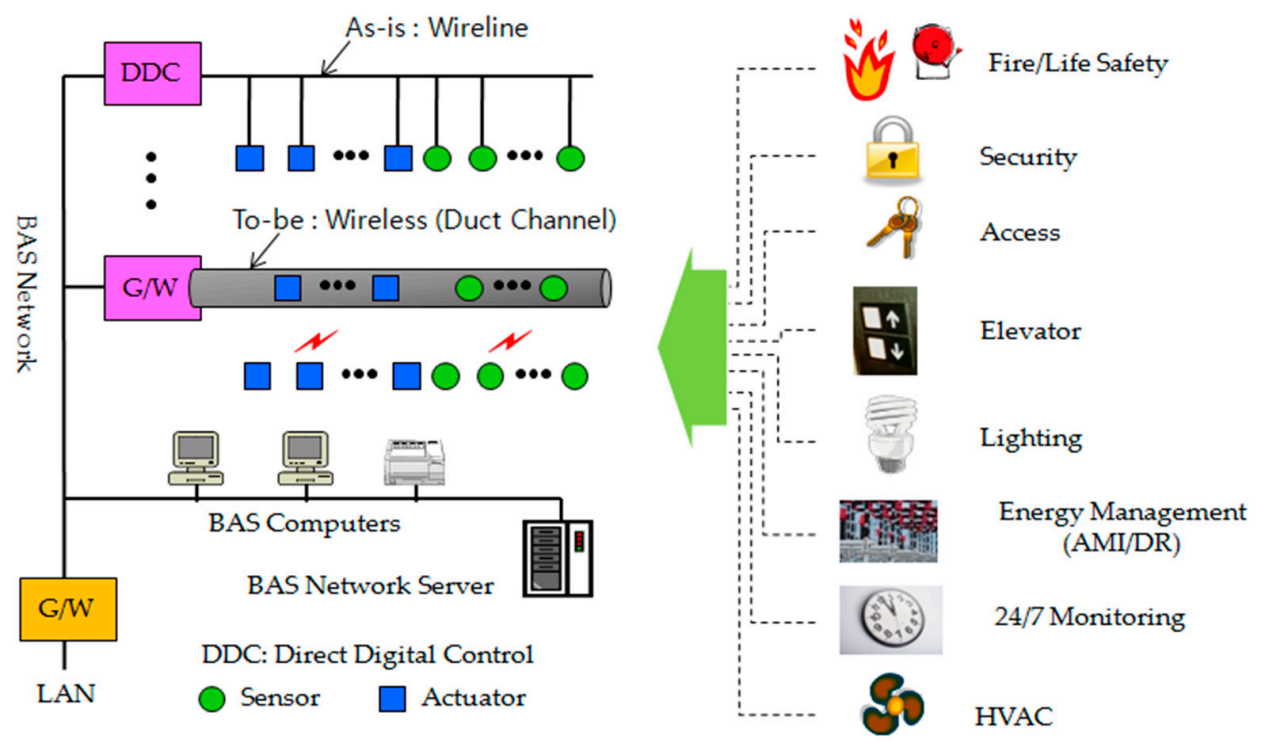

Figure 1. Wireless duct area network (WDAN)-based building's automation systems (BAS) network and services.

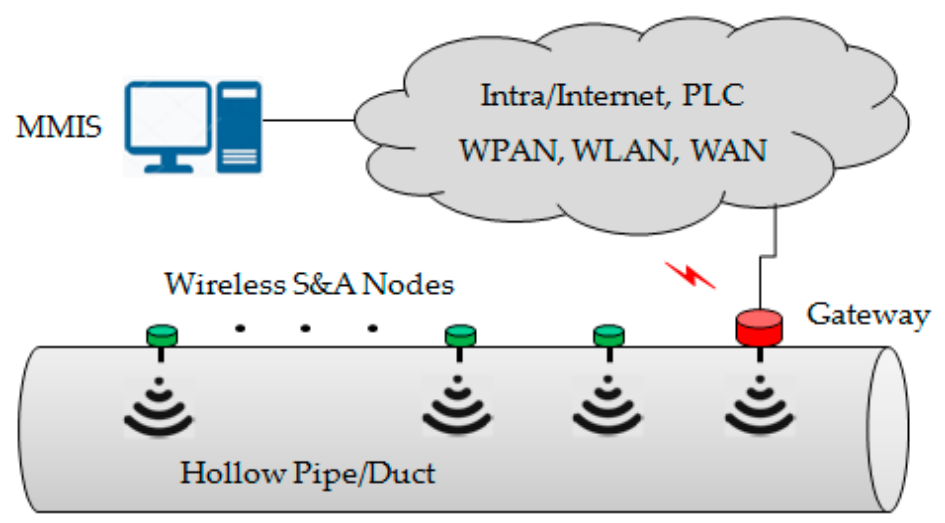

Figure 2. WDAN-based Internet of Things (IoT) network.

\section{Research Methodology}

\subsection{Duct Channel Model}

The hollow metallic pipes, which are modelled as closed time-invariant and electrically shielded waveguides, have a number of important parameters that must considered if they are to be used as an effective communication channel. As they are electrically shielded, inside these pipes, multiple transmitting frequencies can be used without violating interference regulations. In closed waveguides, transverse electric and magnetic fields propagate above the cutoff frequency-below the lowest cutoff frequency propagation is not possible. This frequency is a function of the geometric dimensions of the waveguide. For rectangular pipes, the cutoff frequency is a function of the lengths of the sides of the rectangle [29].

$$
\omega_{c}=c \sqrt{\left(\frac{n \pi}{a}\right)^{2}+\left(\frac{m \pi}{b}\right)^{2}}
$$


where $n, m$ are the mode numbers and $a, b$ are the lengths of the sides of the rectangular pipe. Conversely, the cutoff dimensions of a rectangular pipe for its fundamental mode $\mathrm{TE}_{10}$ can be found using the following mathematical equation:

$$
a_{c}=\frac{\pi c}{\omega}=\frac{c}{2 f}
$$

For circular waveguides, the cutoff frequency depends upon the cross section of the waveguide, which is expressed mathematically as

$$
\omega_{c}=c \frac{x_{01}}{r}=c \frac{2.4048}{r}
$$

where $r$ is the radius of the circular dimension of the waveguide, $c$ is the speed of light and $x_{01}$ is the first root of $J_{0}(r)$, a Bessel function of the first kind of order 1 . For the dominant mode $\mathrm{TE}_{11}$, the cutoff frequency is given by [29]

$$
\omega_{c}=c \frac{x_{11}}{r}=\mathrm{c} \frac{1.8412}{r}
$$

Conversely, the cutoff radius of a circular pipe for its fundamental mode $\mathrm{TE}_{11}$ can be found using the following equation:

$$
r_{c}=c \frac{1.8412}{2 \pi f}
$$

The allocation of ISM radio frequencies is provided according to Article 5 of the ITU Radio Regulations (Edition 2012) [30]. Near or within these ISM bands, various IoT communication networks such as WPAN (ZigBee, Z-Wave, 6LowPAN, Bluetooth), WLAN (WiFi), Non-cellular based LPWA (Low Power Wide Area, SigFox, LoRa) can be deployed. The minimum dimensions of the rectangular and circular pipes capable of acting as waveguides in an industrial environment for the frequency bands of interest are shown in Table 1.

Table 1. Cutoff dimensions for rectangular and circular ducts.

\begin{tabular}{ccc}
\hline $\begin{array}{c}\text { ISM Frequency Band/ } \\
\text { Center Frequency }\left(f_{c}\right)\end{array}$ & $\begin{array}{c}\text { Rectangular Cross-Section } \\
\left(\boldsymbol{a}_{\boldsymbol{c}}, \mathbf{c m}\right) @ f_{c}\end{array}$ & $\begin{array}{c}\text { Circular Cross-Section } \\
\left(\boldsymbol{r}_{c}, \mathbf{c m}\right) @ f_{c}\end{array}$ \\
\hline $902-928 / 915 \mathrm{MHz}$ & 16.4 & 9.6 \\
$2.4-2.5 / 2.45 \mathrm{GHz}$ & 6.1 & 3.6 \\
$5.725-5.875 / 5.8 \mathrm{GHz}$ & 2.6 & 1.5 \\
$24-24.25 / 24.125 \mathrm{GHz}$ & 0.62 & 0.37 \\
\hline
\end{tabular}

As the data presented in Table 1 shows, the standard circular pipes typically used for HVAC ducts, waste collection pipelines, and gas pipelines in industrial environments, which normally have a radius in the order of 15 to $30 \mathrm{~cm}$, should be eminently suitable for use as waveguides for an IoT communication network.

\subsection{Duct Channel Characterization}

\subsubsection{Path Loss and Received Power}

The path loss between communicating antennas depends upon the specific medium through which the signal propagates. In a multipath propagating environment, the path loss is defined by Nikitin and Rao [31] as follows:

$$
P_{L}=\left(\frac{\lambda}{4 \pi d}\right)^{2}\left|1+\sum_{n=1}^{=N} \Gamma_{n} \frac{d}{d_{n}} e^{-j k\left(d_{n}-d\right)}\right|^{2}
$$


where $d$ is the length of the direct ray path, $\Gamma_{n}$ is the reflection coefficient of the nth reflecting object, $d_{n}$ is the length of the $n$th reflected ray path, and $N$ is the total number of reflections. The received signal strength for the multipath propagating medium can be calculated from the transmitted power, transmitter antenna gain, and path loss [31].

$$
P_{r}=P_{t} G_{t} P_{L}
$$

where $P_{r}$ is the power arriving at the receiver, $P_{t}$ is the transmitted power, $G_{t}$ is the transmitter antenna gain, and $P_{L}$ is the path loss. In a cluttered environment, the path loss is usually proportional to $d^{-n}$ [31]. The value of $n$ depends on the propagation environment. In free space, the value of $n$ is equal to 2 and the path loss in $\mathrm{dB}$ is given by [32]

$$
P_{L}=10 \log \frac{P_{t}}{P_{r}}=-10 \log \left[\frac{G_{t} G_{r} \lambda^{2}}{(4 \pi d)^{2}}\right]
$$

where $G_{r}$ is the receiver antenna gain and $d$ is the separation between the transmitter and receiver antenna. The received signal power at the receiver in free space can be calculated by the Friis free space equation [32] or by the equation given by Nikitin and Rao [31], respectively.

$$
P_{r}=\frac{P_{t} G_{t} G_{r} \lambda^{2}}{(4 \pi d)^{2} L}=\frac{P_{t} G_{t}^{2} G_{r}^{2} \lambda^{2} K}{(4 \pi d)^{2}}
$$

where $L$ is the system loss factor in the Friis equation and is not related to propagation. The value of $L$ is greater than or equal to 1 . As defined by Nikitin and Rao [31], $K$ is the backscatter modulation loss and indicates the amount of RF power the receiver is able to convert to the modulated backscatter. It is important to note that in the presence of obstructions $n$ will have a larger value. Due to the many obstructions commonly found in industrial environments, the value of $n$ generally varies between 2 and 3 . As the value of $n$ increases, the path loss also rises and is given by the following equation in $\mathrm{dB}[32]$ :

$$
P_{L}=P_{L}\left(d_{0}\right)+10 n \log \frac{d}{d_{0}}
$$

where $d_{0}$ is a close-in reference distance determined from measurements close to the transmitter. In straight waveguides, the mode loss is linear with distance $d$, but in free space and industrial environments, it is proportional to the power of distance [32]. This indicates that the path loss in pipes and ducts, acting as waveguides, is smaller than it would be in free space. Normally, the path loss in free space is smaller than in an industrial environment. The above analysis thus indicates that the path loss in a pipe waveguide is much smaller than the path loss in a normal industrial environment. Likewise, the power received at the receiver will be lower in an industrial environment compared to either free space or a straight pipe waveguide. A comparison of the received power and path loss is provided in Table 2 for free space, multipath, industrial environment and duct communication. The table clearly shows that path loss is markedly lower in duct communication channels compared with other media and the received power is highest in duct waveguides. However, a more realistic path loss model for individual duct components that includes bends, tees, and wyes is presented by Tonguz et al. [33].

Table 2. Path loss comparison among different propagation channels.

\begin{tabular}{ccccc}
\hline Channel Type & Free Space & Multipath & Industrial & Duct \\
\hline$P_{L} \propto$ & $d^{2}$ & $d^{4}$ & $d^{2 \sim 3}$ & $d^{1 \sim 1.5}$ \\
$P_{r} \propto$ & $1 / d^{2}$ & $1 / d^{4}$ & $1 / d^{2 \sim 3}$ & $1 / d^{1 \sim 1.5}$ \\
\hline
\end{tabular}




\subsubsection{Interference and Fading}

Several standards, including IEEE $802.11 \mathrm{~b} / \mathrm{g} / \mathrm{a}$ WLAN, IEEE 802.15.1-2005 Bluetooth, IEEE 802.15.4 ZigBee, wireless USB, cordless phones, and microwave ovens share the license-free $2.4 \mathrm{GHz}$, industrial, scientific, and medical (ISM) portion of the radio spectrum. The co-existence of these standards in the ISM band often results in mutual interference, degrading the performance and reliability of communication. The interference between these technologies has been extensively investigated in the literature. In an industrial environment, the problem of interference was highlighted by Willig et al. [34] who compared the performance of Bluetooth, WLAN and ZigBee in a typical factory floor environment. In industrial settings, none of the $2.4 \mathrm{GHz}$ ISM-band technologies are able to run at their nominal performance levels because of the seriously adverse radio conditions, primarily interference [35]. Hence, new methods that decrease this mutual interference are urgently needed. Fortunately, inside most industrial environments, we have the opportunity to mitigate interference and fading using HVAC ducts, garbage carrying pipes, and gas pipelines as communication channels.

\section{Experimental Results and Discussion}

\subsection{Experimental Results}

We conducted extensive experiments to test the feasibility of deploying WSN in an industrial environment using in-pipe communication. The purpose of these experiments was to provide proof-of-principle deployment of SA nodes inside a hollow metallic duct in real-world situations. To validate the concept of the effective propagation of a ZigBee network inside hollow metallic pipes, we conducted experiments for two different scenarios. The experimental setup was based on the proposed system architecture shown in Figure 2. In the first setup, ZigBee communication was tested inside a $200 \mathrm{~m}$ long vertical pipe with a diameter of $40 \mathrm{~cm}$ (i.e., a $20 \mathrm{~cm}$ radius) typical of those installed to carry garbage in residential apartments in Seoul. In the second set of experiments, the ZigBee communication was tested inside a $30 \mathrm{~cm}$ diameter (i.e., $15 \mathrm{~cm}$ radius), $90 \mathrm{~m}$ long straight garbage carrying pipe. Figure 3 shows snapshots of the actual setup used for testing ZigBee communication in the garbage carrying pipe.

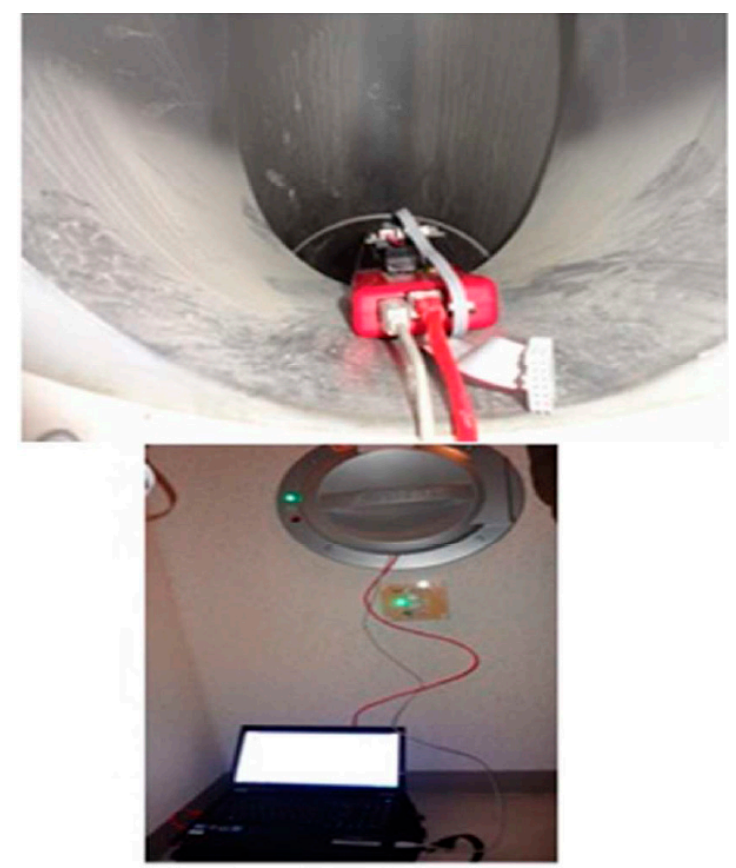

Figure 3. Experimental scenario for in-pipe wireless communications. 
For these experiments, we used Ember EM357-integrated ZigBee Radio Control Modules (RCM) [36] as the transmitting and receiving WSN nodes. The general specifications of a ZigBee RCM are given in Table 3.

Table 3. ZigBee Radio Control Modules (RCM) general specifications.

\begin{tabular}{cc}
\hline Parameter & Description \\
\hline Processor & 32-bit Arm Cortex M3 \\
Radio Chip & $2.4 \mathrm{GHz}$ IEEE $802.15 .4-2003$ transceiver \\
Data Rate & $250 \mathrm{kbps}$ \\
Modulation Format & DSSS (OQPSK) \\
Transmit Power & $5 \mathrm{dBm}, 8 \mathrm{dBm}$ in boost mode \\
Receive Sensitivity & $-102 \mathrm{dBm}(1 \%$ PER, 20 byte packet $)$ \\
\hline
\end{tabular}

As noted earlier, ZigBee operates in the $2.4 \mathrm{GHz}$ ISM band. Readings from the received signal strength indicator (RSSI) were recorded by the Ember Insight Desktop as a function of the separation distance between the transmitter and receiver node. During the experiment, the ZigBee coordinator (ZC) was placed on the first floor of the residential apartment building inside the vertical pipe of a conventional wired communication based automatic waste collection system (AWCS). A series of sensor nodes (normally a ZigBee end device (ZED), although in some cases a ZigBee router (ZR) was used) were placed at different distances from the ZC, and the resulting RSSI was measured between ZC and ZED through the pipe.

A comparison of the signal fade in free space compared to signal fade in a pipe/duct waveguide, based on repeated experiments, is shown in Figure 4. The instantaneous variation in signal power is calculated to be $7 \mathrm{dBm}$ in free space, while in a pipe/duct waveguide, it fell dramatically to just $1 \mathrm{dBm}$. These results demonstrate that the proposed waveguides outperform free-space propagation in terms of both fading and interference.

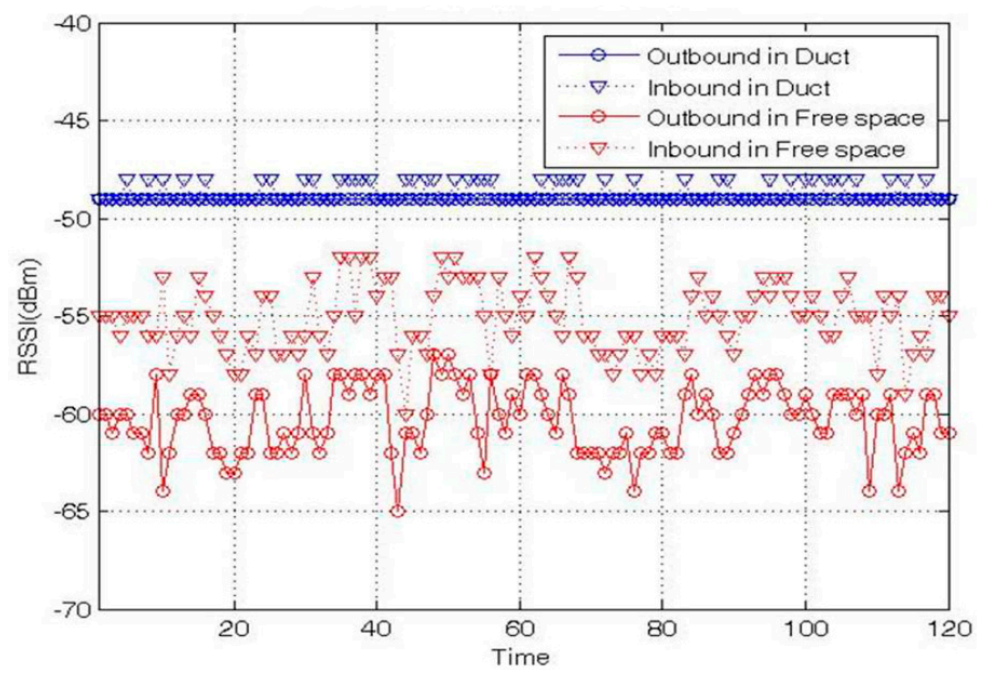

Figure 4. Deviation of received signal strength indicator (RSSI) in duct and free space.

Figure 5a shows the results obtained for the RSSI measurements as a function of separation distance $\mathrm{d}$ between the coordinator and the end device. In the graph, the $\mathrm{x}$-axis represents the separation distance $\mathrm{d}$ between ZC and ZED, while the $\mathrm{y}$-axis shows the RSSI measurements. Here, the red symbols denote the signal strength from ZED to ZC (inbound), while the blue symbols indicate the signal strength from ZC to ZED (outbound). The RSSI measurement was initially quite good but dropped gradually with increasing separation distance d. At d = $192 \mathrm{~m}$ (64th floor), the RSSI had dropped to $-74 \mathrm{dbm}$ with a one hop star topology with no packet transmission errors, which represents 
reliable transmission. Based on the ZigBee specifications [36], we estimate that even allowing a $30 \mathrm{dBm}$ margin it should be possible to provide a further $60 \mathrm{~m} \sim 70 \mathrm{~m}$ coverage with only one hop inside this duct. Figure $5 \mathrm{~b}$ depicts the results for the RSSI measurements with respect to the separation distance $\mathrm{d}$ at different locations inside the pipe. The graph shows that at a separation distance of $d=84 \mathrm{~m}$ (the building's 28th floor), the RSSI was only $-35 \mathrm{dbm}$, with no packet loss. These results demonstrate that compared to free space propagation, hollow pipe/duct waveguides can provide a safe, reliable, and interference-free channel, greatly expanding the coverage of WSN communication networks.

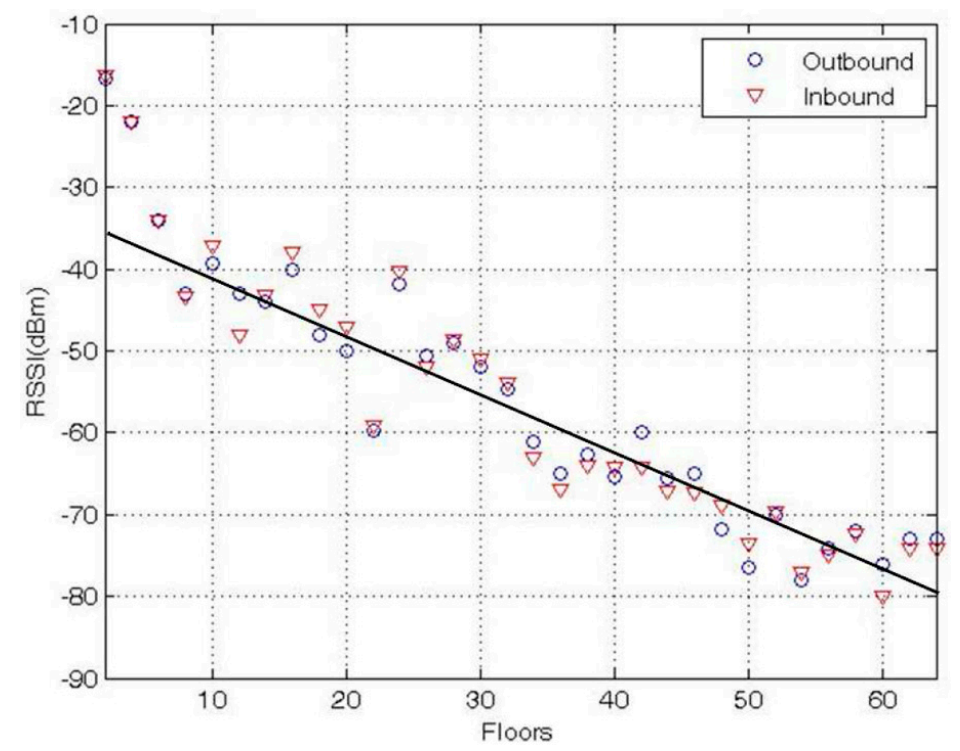

(a)

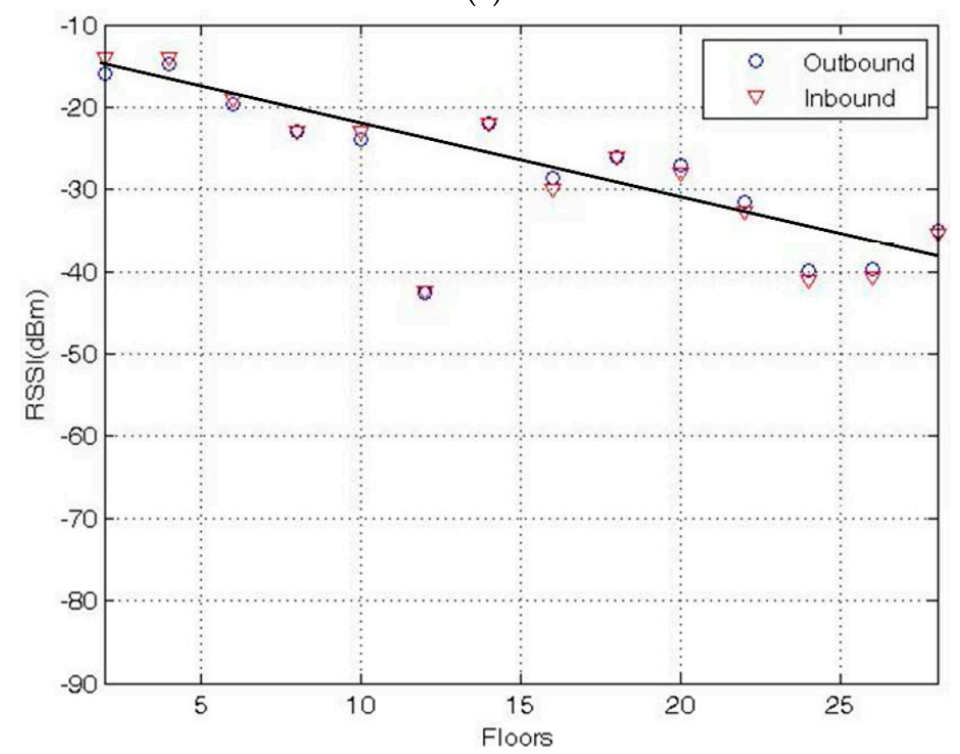

(b)

Figure 5. (a) RSSI in 64-story mixed apartment building ( $40 \mathrm{~cm}$ diameter pipe); (b) RSSI in 28-story apartment building ( $30 \mathrm{~cm}$ diameter pipe).

\subsection{Proposed WDAN Architecture}

The aim of this research has been to replace the complex web of wires currently used with a ZigBee network by instead utilizing in-pipe communication channels inside electrically noisy industrial environments. In the harsh environments commonly found in industrial settings, ZigBee offers numerous benefits, including remote monitoring of devices and automatically controlling them via its 
wireless mesh network (WMN). The ZigBee WMN, which is generally referred to as a personal area network (PAN), mainly consists of two types of device nodes: full function devices (FFD) and reduced function devices (RFD). An FFD device in a ZigBee PAN can take one of two roles: either a ZigBee coordinator (ZC) or a ZigBee router (ZR). The ZC, which is unique within the network, is usually called the PAN coordinator and is responsible for starting and maintaining the network. The $Z R$, which is also an FFD, has the same routing capabilities as the ZC but can only join an existing network, and never form it. All ZRs in the network are declared "joined and authenticated" after a successful authentication process. An RFD called a ZigBee end device (ZED) is a passive node with no routing capability and can be operated as a sleepy device powered by batteries.

Figure 6 illustrates a WDAN deployment in a simple HVAC system to support building automation. In each room of the building, various sensor nodes (temperature/humidity, air quality, RFID reader, illumination level, fire, intrusion, presence, thermostat/switches, meters, blinds, etc.) and actuator nodes (light dimming, AC, etc.) can be connected to the gateway via a router installed at the diffuser. Applying our proposed WDAN based HVAC system will provide more efficient control and thus achieve further energy savings.

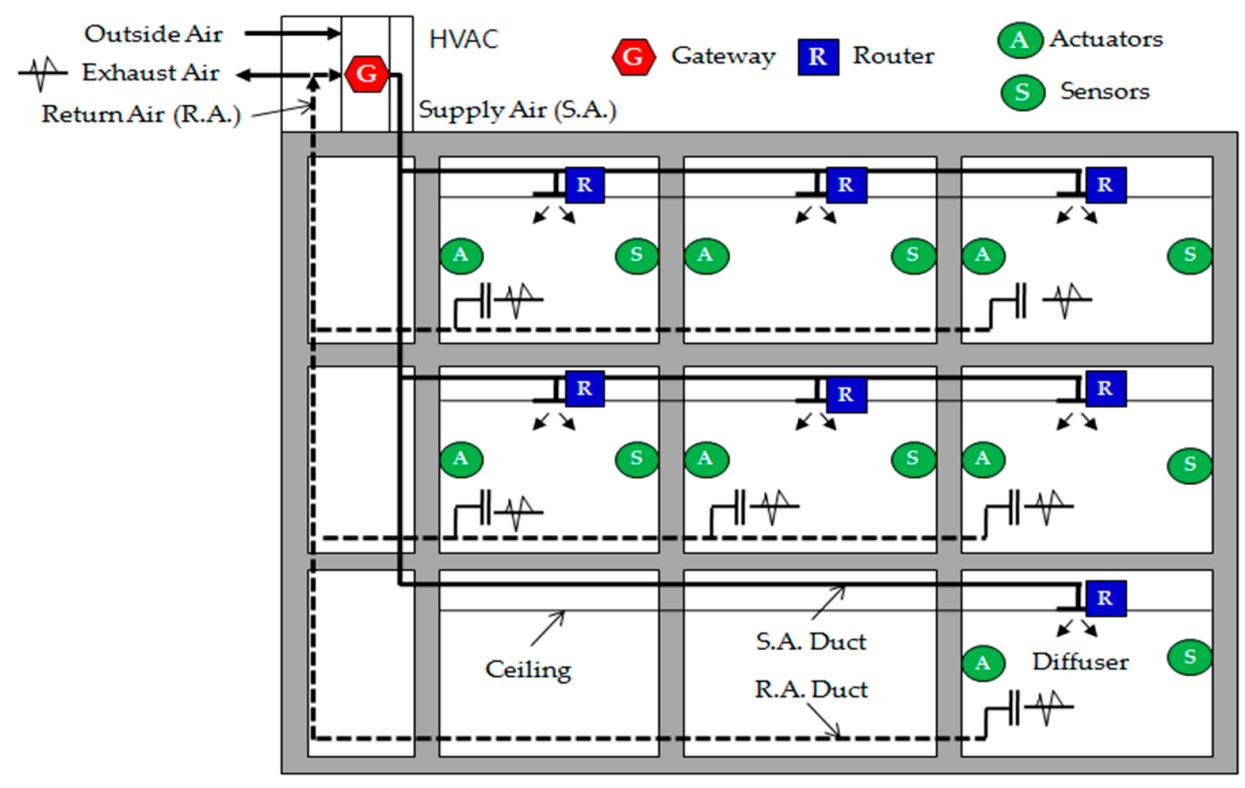

Figure 6. A proposed WDAN architecture for BAS.

\section{Conclusions}

This paper presents an innovative architecture for deploying wireless sensors inside existing pipes to form a WDAN using a building's ducts as a channel for signal propagation. The design is appropriate for skyscrapers, commercial buildings, and industrial environments utilizing the HVAC ducts, gas pipeline networks and waste collection pipes, which are essentially electromagnetic waveguides, that are already incorporated into existing building infrastructure elements. The theoretical discussion indicated that these duct waveguides should provide reliable and interference-free propagation channel compared to free space, multipath and industrial environments for wireless communication. This was confirmed experimentally by installing a ZigBee communication network inside hollow ducts of a type commonly used to dispose of garbage; the results obtained clearly validated the successful deployment of WSN inside these ducts. The RSSI results indicate that WSN can be successfully extended to $250 \mathrm{~m}$ inside a duct with one hop at a frequency of $2.4 \mathrm{GHz}$. This confirms that WDAN can indeed serve as a cost-effective, reliable, sustainable and user-friendly control and automation network that can be applied for a wide range of applications, including pipe inspections, leak detection, and condition monitoring in commercial, residential, and industrial environments. 
From a sustainability point of view, this proposed WDAN system provides a number of economic, environmental, and social advantages that will help make intelligent buildings more sustainable, as depicted in Figure 7. First of all, a wireless duct network reduces the initial cost of providing monitoring systems and communication networks at the construction stage as well as being easily retrofitted to existing buildings. To install conventional wired communication systems in large buildings, long cables must be connected to individual IoT components, and multiple wireless routers must be installed to send or receive the signals propagated across the network. These are best installed during the construction stage of building project, though they can be retrofitted, albeit at a considerable cost. Periodic maintenance is also required to maintain the system's reliability. In contrast, the WDAN system proposed here does not incur the major expense involved in installing expensive coaxial communication cables and multiple wireless routers in a building. It is also easier to conduct corrective maintenance activities because there are no complex communication cables and routers involved. These advantages make WDAN far more cost effective than existing wired networks.

Second, WDAN systems consume less electricity, as they require fewer pieces of communication equipment such as network cables, routers, and so forth. Also, a wireless sensor network that uses an existing duct system has greater extendibility, enabling users to easily add extra sensors or actuators to control new equipment. This will greatly aid future efforts to upgrade a building's sustainable credentials by facilitating the installation of new, more energy efficient technologies as they become available, thus reducing its energy footprint and lowering $\mathrm{CO}_{2}$ emissions via advanced building MEP/HVAC systems.

Third, based on these economic and environmental advantages, WDAN systems will enable facility managers to provide a wide range of services as well as control important environmental conditions such as temperature, humidity, air quality, security, and fire protection, thus improving the living/working conditions of the building's occupants. The precise control of indoor conditions made possible by tracking temperature and humidity in real time will enhance the health and comfort of residents and visitors alike. In the event of an emergency, such as a fire, terrorism threat, or disaster, an IBS system would prevent the flames from spreading across the entire building by controlling the diffusers, dampers, and fans that are integral parts of such duct systems. Wireless sensor networks installed in a building's ducts would also provide real-time security for residents by sensing trespassers or intruders in the building. Overall, WDAN could make a major contribution to the sustainability of intelligent buildings, supporting the economic, environmental, and social dimensions of sustainability.

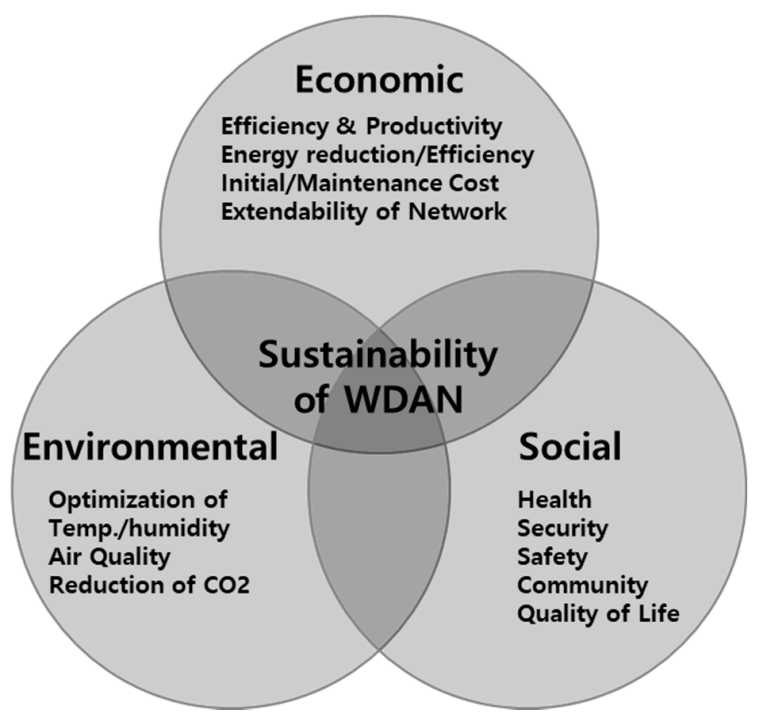

Figure 7. Sustainability of WDAN. 
Author Contributions: Conceptualization, H.Y., J.L. and Y.A.; Investigation, Y.A.; Methodology, H.Y., B.K., J.L. and C.L.; Project administration, J.L., Y.A. and C.L.; Resources, B.K.; Supervision, H.Y.; Validation, H.Y.; Writing-original draft, H.Y. and J.L.; Writing-review \& editing, H.Y., J.L. and C.L.

Funding: This research received no external funding.

Acknowledgments: This work was supported by the Korean Institute of Energy Technology Evaluation and Planning (KETEP) and the Ministry of Trade, Industry \& Energy (MOTIE) of the Republic of Korea (No. 20172010000370) and a National Research Foundation of Korea Grant from the Korean Government (MSIT; the Ministry of Science and ICT) (NRF-2016M1A5A1901769) (KOPRI-PN18081).

Conflicts of Interest: The authors declare no conflict of interest.

\section{References}

1. Sodhro, A.H.; Pirbhulal, S.; Sangaiah, A.K.; Lohano, S.; Sodhro, G.H.; Luo, Z. 5G-Based Transmission Power Control Mechanism in Fog Computing for Internet of Things Devices. Sustainability 2018, 10, 1258. [CrossRef]

2. Fallah, S.N.; Deo, R.C.; Shojafar, M.; Conti, M.; Shamshirband, S. Computational Intelligence Approaches for Energy Load Forecasting in Smart Energy Management Grids: State of the Art, Future Challenges, and Research Directions. Energies 2018, 11, 596. [CrossRef]

3. Pooranian, Z.; Abawajy, J.H.; Vinod, P.; Conti, M. Scheduling Distributed Energy Resource Operation and Daily Power Consumption for a Smart Building to Optimize Economic and Environmental Parameters. Energies 2018, 11, 1348. [CrossRef]

4. Cao, X.; Chen, J.; Xiao, Y.; Sun, Y. Building-Environment Control With Wireless Sensor and Actuator Networks: Centralized Versus Distributed. IEEE Trans. Ind. Electron. 2010, 57, 3596-3605.

5. Nguyen, N.H.; Tran, Q.T.; Leger, J.M.; Vuong, T.P. A real-time control using wireless sensor network for intelligent energy management system in buildings. In Proceedings of the 2010 IEEE Workshop on Environmental Energy and Structural Monitoring Systems (EESMS), Taranto, Italy, 9 September 2010; pp. 87-92.

6. Junnila, S.; Kailanto, H.; Merilahti, J.; Vainio, A.M.; Vehkaoja, A.; Zakrzewski, M.; Hyttinen, J. Wireless, Multipurpose In-Home Health Monitoring Platform: Two Case Trials. IEEE Trans. Inf. Technol. Biomed. 2010, 14, 447-455. [CrossRef] [PubMed]

7. Munir, A.; Gordon-Ross, A. An MDP-Based Dynamic Optimization Methodology for Wireless Sensor Networks. IEEE Trans. Parallel Distrib. Syst. 2012, 23, 616-625. [CrossRef]

8. Chen, J.; Cao, X.; Cheng, P.; Xiao, Y.; Sun, Y. Distributed Collaborative Control for Industrial Automation with Wireless Sensor and Actuator Networks. IEEE Trans. Ind. Electron. 2010, 57, 4219-4230. [CrossRef]

9. Yoo, S.E.; Chong, P.K.; Kim, D.; Doh, Y.; Pham, M.L.; Choi, E.; Huh, J. Guaranteeing Real-Time Services for Industrial Wireless Sensor Networks With IEEE 802.15.4. IEEE Trans. Ind. Electron. 2010, 57, 3868-3876. [CrossRef]

10. Sheikh, A.U.H.; Al-Moellam, Y.D. Wireless Networking for Monitoring and Control System of a Steel Plant. Wirel. Pers. Commun. 2011, 66, 1-18. [CrossRef]

11. Cheong, P.; Ka-Fai, C.; Ying-Hoi, L.; Sut-Kam, H.; Iam-Keong, S.; Kam-Weng, T. A ZigBee-Based Wireless Sensor Network Node for Ultraviolet Detection of Flame. IEEE Trans. Ind. Electron. 2011, 58, 5271-5277. [CrossRef]

12. Sri Jahnavi, V.; Fayaz Ahmed, S. Smart Wireless Sensor Network for Automated Greenhouse. IETE J. Res. 2015, 61, 180-184. [CrossRef]

13. Gungor, V.C.; Hancke, G.P. Industrial Wireless Sensor Networks: Challenges, Design Principles, and Technical Approaches. IEEE Trans. Ind. Electron. 2009, 56, 4258-4265. [CrossRef]

14. Anastasi, G.; Conti, M.; Di Francesco, M. A comprehensive analysis of the MAC unreliability problem in IEEE 802.15. 4 wireless sensor networks. IEEE Trans. Ind. Informat. 2011, 7, 52-65. [CrossRef]

15. Bertocco, M.; Gamba, G.; Sona, A.; Vitturi, S. Experimental Characterization of Wireless Sensor Networks for Industrial Applications. IEEE Trans. Instrum. Meas. 2008, 57, 1537-1546. [CrossRef]

16. Nikitin, P.V.; Stancil, D.D.; Cepni, A.G.; Tonguz, O.K.; Xhafa, A.E.; Brodtkorb, D. Propagation model for the HVAC duct as a communication channel. IEEE Trans. Antennas Propag. 2003, 51, 945-951. [CrossRef]

17. Xhafa, A.E.; Tonguz, O.K.; Cepni, A.G.; Stancil, D.D.; Nikitin, P.V.; Brodtkorb, D. On the capacity limits of HVAC duct channel for high-speed Internet access. IEEE Trans. Commun. 2005, 53, 335-342. [CrossRef] 
18. Calvo, F.; Moreno, B.; Zamorano, M.; Szanto, M. Environmental diagnosis methodology for municipal waste landfills. Waste Manag. 2005, 25, 768-779. [CrossRef] [PubMed]

19. Fan, X.; Zhu, M.; Zhang, X.; He, Q.; Rovetta, A. Solid waste collection optimization considering energy utilization for large city area. In Proceedings of the International Conference on Logistics Systems and Intelligent Management, Harbin, China, 9-10 January 2010; pp. 1905-1909.

20. Suryadevara, N.K.; Mukhopadhyay, S.C.; Kelly, S.D.; Gill, S.P. WSN-Based Smart Sensors and Actuator for Power Management in Intelligent Buildings. IEEE Trans. Mechatron. 2015, 20, 564-571. [CrossRef]

21. Lai, Y.X.; Rodrigues, J.J.; Huang, Y.M.; Wang, H.G.; Lai, C.F. An Intercommunication Home Energy Management System with Appliance Recognition in Home Network. Mob. Netw. Appl. 2012, 17, 132-142. [CrossRef]

22. Langhammer, N.; Kays, R. Performance Evaluation of Wireless Home Automation Networks in Indoor Scenarios. IEEE Trans. Smart Grid 2012, 3, 2252-2261. [CrossRef]

23. Yi, P.; Iwayemi, A.; Zhou, C. Developing ZigBee Deployment Guideline under WiFi Interference for Smart Grid Applications. IEEE Trans. Smart Grid 2011, 2, 110-120. [CrossRef]

24. Bhatt, J.; Verma, H.K. Design and Development of Wired Building Automation Systems. Energy Build. 2015, 103, 369-413. [CrossRef]

25. Nikitin, P.V.; Stancil, D.D.; Tonguz, O.K.; Xhafa, A.E.; Cepni, A.G.; Brodtkorb, D. Impulse response of the HVAC duct as a communication channel. IEEE Trans. Commun. 2003, 51, 1736-1742. [CrossRef]

26. Henty, B.E. Advanced System Design of In-Building Wireless Communication Networks Using Ventilation Ducts. Ph.D. Thesis, Carnegie Mellon University, Pittsburgh, PA, USA, 2006.

27. Rashid, B.; Rehmani, M.H. Applications of wireless sensor networks for urban areas: A survey. J. Netw. Comput. Appl. 2016, 60, 192-219. [CrossRef]

28. Sirsikar, S.; Anavatti, S. Issues of Data Aggregation Methods in Wireless Sensor Network: A Survey. Procedia Comput. Sci. 2015, 49, 194-201. [CrossRef]

29. Hunter, I.C. Theory and Design of Microwave Filters; The Institution of Engineering and Technology: London, UK, 2001; Volume 48.

30. International Telecommunication Union (ITU). CHAPTER II-Frequencies, Article 5 Frequency allocations, Section IV-Table of Frequency Allocation. In Radio Regulations; ITU: Geneva, Switzerland, 2016.

31. Nikitin, P.V.; Rao, K. Antennas and propagation in UHF RFID systems. In Proceedings of the IEEE International Conference on RFID, Las Vegas, NV, USA, 16-17 April 2008; pp. 277-288.

32. Rappaport, T.S.; Online, S.B. Wireless Communications: Principles and Practice, 2nd ed.; Prentice Hall: Upper Saddle River, NJ, USA, 1996.

33. Tonguz, O.K.; Xhafa, A.E.; Stancil, D.D.; Cepni, A.G.; Nikitin, P.V.; Brodtkorb, D. A simple path-loss prediction model for HVAC systems. IEEE Trans. Veh. Technol. 2004, 53, 1203-1214. [CrossRef]

34. Willig, A.; Matheus, K.; Wolisz, A. Wireless Technology in Industrial Networks. Proc. IEEE 2005, 93, 1130-1151. [CrossRef]

35. Zigbee Alliance. ZigBee Specification; ZigBee Document 053474r13; Zigbee Alliance: Davis, CA, USA, 2006; pp. 344-346.

36. EM351/EM357. Available online: https://www.silabs.com/documents/public/data-sheets/EM35x.pdf (accessed on 15 July 2018).

(C) 2018 by the authors. Licensee MDPI, Basel, Switzerland. This article is an open access article distributed under the terms and conditions of the Creative Commons Attribution (CC BY) license (http://creativecommons.org/licenses/by/4.0/). 